\title{
ALGUNAS PRUEBAS DE HIPÓTESIS ESTADÍSTICAS CON SPSS
}

Responsable: Lic. Luis López Puycan Miembro: Lic. Javier Lozano Marreros

\section{RESUMEN}

La presente investigación tiene como objetivo general determinar algunas pruebas de hipótesis estadisticas con SPSS. Que se utilizaron con el sofwart : la prueba de bondad de ajuste de kolmogorov- smirnov aplicando pruebas no paramétricas y K-S de una muestra, K muestras independientes, la prueba $\mathrm{h}$ de kruskall- wallis para $\mathrm{k}$ muestras independientes, prueba de la mediana, prueba para varias muestras relacionadas usando la prueba de Friedman W de Kendall, la prueba de Chi cuadrado, prueba de la binomial así, como también para la estadistica paramétrica se utilizo la prueba t para muestras relacionadas, comparar medias, prueba t para una muestra, prueba t para muestras independientes y análisis de varianza.

\section{ABSTRACT}

The present investigation has as general objective, determine some tests of statistical hypothesis with SPSS.

The referred investigation in the parametric and not parametric statistic being in this some tests of statistical hypothesis that were used with the software was the following test: the test of kindness of kolmogorovSmirnov adjustment applying non parametric tests and $\mathrm{k}$-s of a sample, $\mathrm{k}$ independent samples, the test $\mathrm{H}$ of Kruskall - wallis for $k$ independent samples, proves of the medium one, it proves for several related samples using the friedman test kendall $w$, the test of square chi, proves of the binomial one as well as for the parametric statistic you use. the test $t$ for related samples, to compare average, $t$ proves for a sample, $t$ proves for independent samples and variance analysis

\section{INTRODUCCION}

Pueden presentarse en la práctica situaciones en las que exista una teoría preconcebida relativa a la característica de la población sometida a estudio. Tal sería el caso, por ejemplo si pensamos que un tratamiento nuevo puede tener un porcentaje de mejoría mayor que otro estándar, o cuando nos planteamos si las personas de las distintas comunidades tienen la misma altura. Este tipo de circunstancias son las que nos llevan al estudio de la parte de la Estadística Inferencial que se recoge bajo el título genérico de Contraste de Hipótesis. Implica, en cualquier investigación, la existencia de dos teorías o hipótesis implícitas, que denominaremos hipótesis nula e hipótesis alternativa, que de alguna manera reflejarán esa idea a priori que tenemos y que pretendemos contrastar con la "realidad". De la misma manera aparecen, implícitamente, diferentes tipos de errores que podemos cometer durante el procedimiento. No podemos olvidar que, habitualmente, el estudio y las conclusiones que obtengamos para una población cualquiera se habrán apoyado exclusivamente en el análisis de sólo una parte de ésta. De la probabilidad con la que estemos dispuestos a asumir estos errores.La Estadística es una herramienta fundamental que se aplica en todas las áreas profesionales como en la ingeniería, en la administración, en la ciencia de la salud, etc. La estadística trata de la selección, análisis y uso de datos con el fin de resolver problemas. Consecuentemente, algunos conocimientos de estadística le serán de utilidad a la población en general, pero en particular, el conocimiento estadístico será vital para la investigación.. Por lo que es necesario e indispensable el uso de software estadístico donde se aplique algunas pruebas de hipótesis estadística con SPSS y asi minimizar el tiempo de poder resolver los problemas de algunos tópicos de la estadística. Desarrollamos en esta parte los contrastes de hipótesis para los parámetros más usuales en la estadística paramétrica y no paramétrica .

\section{OBJETIVOS}

- Desarrollar Algunas pruebas de hipótesis estadísticas con SPSS.

- Determinar el contraste de la prueba Kolmogorov* Sminorv.

- Determinar el contraste de la prueba binomial para muestra.

- Determinar el contraste de la prueba ChiCuadrado.

\section{Material}

Para la realización de este trabajo se hizo un estudio inferencial con ejemplos bibliográficos ya sean datos cuantitativos 0 cualitativos 


\section{Métodos}

Se utilizaron algunas pruebas de hipótesis paramétricas y no paramétricas.

Se utilizará el uso del software estadistico SPSS 15 para el procesamiento de datos Resultados:

Problema 1: Se efectuaron mediciones del nivel de glucosa en sangre de 36 hombres adultos en ayuno, no obesos y aparentemente sanos.

\section{Estas mediciones se muestran en la Tabla 1.}

Se pretende saber si es posible concluir que tales datos no pertenecen a una población que sigue una distribución normal.

Tabla 01: Valores de glucosa en sangre $(\mathrm{mg} / 100 \mathrm{ml})$, obtenidos en ayunas, de 36 individuos no obesos y aparentemente sanos.

\begin{tabular}{|l|l|l|l|l|l|}
\hline 75 & 92 & 80 & 80 & 84 & 72 \\
84 & 77 & 81 & 77 & 75 & 81 \\
80 & 92 & 72 & 77 & 78 & 76 \\
77 & 86 & 77 & 92 & 68 & 87 \\
68 & 78 & 92 & 68 & 80 & 81 \\
87 & 76 & 80 & 87 & 77 & 86 \\
\hline
\end{tabular}

Prueba de Kolmogorov-Smirnov para una muestra:

$\mathrm{N}=36$ Media 80,08

Parámetros normales $(a, b)$ desviación típica 6,199

Diferencias más extremas :

Absoluta 0,163

Positiva 0,163

Negativa $-0,095$

Z de Kolmogorov-Smirnov 0,981

Sig.asintót.(bilateral) 0,291.

a: La distribución de contraste es la normal

b: Se han calculado a partir de los datos

La diferencia máxima en un valor absoluto de la frecuencia observada y esperada es 0,163 . Por otra parte, en la tabla de valores de kolmogorov-Smirnov para el nivel de significación del $5 \%$ y $n=36$ se encuentra el valor crítico 0,221 . Dado que $0,163<0,221$, debemos aceptar la hipótesis nula de que es normal la población de la cual se ha obtenido la muestra .

Próblema 2: En una clínica se quiere contrastar si existe diferencia significativa entre tres tipos de calmantes fuertes. Con este fin se suministran las drogas independientes a pacientes comparables midiéndose el tiempo en segundos desde la inyección de la droga hasta la desaparición del dolor, obteniéndose la siguiente tabla:

Drogas:

A: $30,35,60,61,42$

B: $41,32,70,56,80,43$

C: $62,57,35,42,78$.

¿Se puede afirmar con un nivel de significación de 0.05 que las tres drogas producen en términos medio los mismos efectos?.

Se trata de contrastar si hay homogeneidad en los efectos de tres drogas suministradas independiente.

Prueba de kruskal-Wallis

Estadisticos de contraste $(a, b)$

Tiempo

Chi-cuadrado 0,973

Gl. 2

Sig.asintót. 0,615.

a:Prueba de kruskall-Wallis

b:variable de agrupación droga

Estadistico de contraste (b)

$\mathrm{N}=16$

Mediana 49,50

Chi-cuadrado 0,4000 (a)

GI 2

Sig.asintót. 0,819

a: 6 casillas $(100 \%)$ tienen frecuencias esperadas menores que 5 , la frecuencia de casilla esperada minima es 2,5 .

\section{b:variable de agrupación droga}

Se observa $p$-valor mayores que alfa $=0,05$ para los contrastes, lo que permite aceptar que los efectos son homogéneos, es decir, no existen diferencias significativas entre los tres tipos de calmantes.

Problema 3: Se tomaron muestras de un medio bacteriano que se homogeneizaron y disolvieron. Las disoluciones, de tres grupos, fueron sometidas a la acción de la tetraciclina, la penicilina y una sulfamida. La concentración de bacterias, medida por la densidad óptica registrada por un espectrofotómetro, fue la siguiente:

Tetraciclina: $0,251,0,250,0,249,0,258,0,258$

Penicilina: 0,247, $0,246,0,250,0,240,0,242$

Sulfamida: $0,228,0,236,0,240,0,236,0,230$

Se trata de contrastar si los tratamientos, que pueden ser dependientes porque provienen del mismo medio bacteriano, son o no homogéneos basandose en las tres muestras de que disponemos:

Prueba de Friedman

Rangos

Rango promedio

Sulfamida 1.00

Tetraciclina 2,83

Penicilina 2,17

Estadisticos de contraste (Prueba de Friedman)

$\mathrm{N}=6$ Chi-cuadrado 10,333

gl 2

Sig. Asintót. 0,006

Prueba w de Kendall

Rangos

Rango promedio

Sulfamida 1,00 
Tetraciclina 2,83

Penicilina 2,17

Estadisticos de contraste

$\mathrm{N}=6$

W de Kendall(a) 0,861

Chi-cuadrado 10,33

GI. 2

Sig.asintót. 0,006

a:Coeficiente de concordancia de Kendall

Se observa que el $\mathrm{p}$-valor menor que alfa= 0,05 para los contrastes, lo que permite rechazar que los tratamientos son homogéneos, es decir, existen diferencias entre los tratamientos.

Problema 4: Se clasifican 40 varones según el color del cabello y de los ojos, obteniéndose los siguientes resultados estudiar si existe dependencia entre el color del cabello y el color de los ojos, que se muestra la Tabla 2.

Se trata de estudiar la independencia entre dos variables cualitativas cuyos datos vienen dados en forma de tabla de contingencia. Por lo tanto, utilizaremos el teste de chi-cuadrado para la independencia

Tabla 02:

\begin{tabular}{|l|l|l|l|l|l|}
\hline \multirow{2}{*}{ Ojos } & \multicolumn{5}{c|}{ Cabellos } \\
\cline { 2 - 6 } & Rubio & Castaño & Negro & Rojo & Total \\
\hline Azul & 6 & 2 & 3 & 1 & 12 \\
\hline $\begin{array}{l}\text { Gris o } \\
\text { verde }\end{array}$ & 4 & 5 & 4 & 2 & 15 \\
\hline Pardo & 1 & 3 & 6 & 3 & 13 \\
\hline Total & 11 & 10 & 13 & 6 & 40 \\
\hline
\end{tabular}

Varones:

Prueba de chi-cuadrado (a) 9,8

GI. 11

\section{Sig. Asintót. 0,548}

a:12 casillas (100\%) tiene frecuencias esperadas menores que 5 . La frecuencia de casilla esperada mínima es 3,3

Se concluye que $\mathrm{p}$-valor es mayor que alfa $=$ 0,01 indicando que hay independencia entre color de cabellos y de ojos con un nivel de confianza del $99 \%$.

Problema 5: Se desea comparar la calidad de dos nuevas clases de trigo. Para ello, se toman 10 fincas al azar, plantando en cada una de ellas y en dos partes distintas ambas clases. La producción en lasa fincas fue la siguiente:

Clase A: $57,49,60,55,57,48,50,61,52,56$

Clase B: $55,48,58,56,54,48,52,56,50,58$

A) ¿podemos aceptar que la producción es la misma para ambas clases de trigo a un nivel de significancia del $5 \%$, suponiendo distribución normal bivariante?

B)hallar intervalos de confianza para la media de las diferencias de producciones de ambas clases de trigo

Realizaremos el contraste de hipótesis de igualdad de medias teniendo en cuenta que las dos muestras son dependientes, porque cada producción está tomada en la misma finca y depende de las caracteristicas del terreno; por lo tanto usaremos muestras pareadas para comparar medias pruebaT para muestras relacionadas en spss

Prueba T

Estadísticos de muestras relacionadas

Clase A

Media $=54,50$ desviación típica $=4,552$ error tipico de la media $1,440 \mathrm{~N}=10$

Clase B

Media $=53,50$ desviación típica $=3,808$ error típico de la media $1,204 \mathrm{~N}=10$

Correlación de muestras relacionadas

Clase $A$ y Clase $B N=10$ correlación $=0,869$ sig. $=0,001$

Prueba de muestras relacionadas

Clase Ay clase B .Diferencias relacionadas

Media $=1,00$ desviación típica $=2,261$ error típico de la media 0,715 .

intervalo de confianza del $95 \%$ para la diferencia

Inferior $=-0,617$, superior $=2,617, \mathrm{t}=1,399$, gl. $=9$, sig. (bilateral $)=0,915$.

Se observa que resulta que la diferencia de producciones medias para ambas clases de trigo no es significativa al $95 \%$ ( $p$-valor del contraste de diferencia de medias mayor que alfa $=0,05)$ y lógicamente el intervalo de confianza para dicha diferencia -0,617, 2,617 contiene el valor cero. Se puede concluir que las producciones son iguales en media para ambas clases de trigo.

Problema 6: Una central de productos lácteos recibe diariamente la leche de dos grupos granjas $\mathrm{X}$ e $\mathrm{Y}$. Deseando estudiar la calidad de los productos recibidos se extraen dos muestras, una de cada granja( independientes ), y se analiza el contenido de materia grasa, obteniéndose los siguientes resultados:

$\mathrm{X}$ :

$0,32,0,29,0,30,0,28,0,33,0,31,0,30,0,29,0,33$ $, 0,32,30,0,29$

Y:

$0,28,0,30,0,32,0,29,0,31,0,29,0,33,0,32,0,29$

$, 0,320,31,0,29,0,32,0,31,0,32,0,33$

A) Al nivel de confianza del $99 \%$, determinar un intervalo de confianza para la diferencia de medias, suponiendo normal la variable estudiada ( contenido de materia grasa ) en ambas poblaciones independientes

B) Realizar el contraste de hipótesis de igualdad de medias para alfa $=0,01$

Se realizará una prueba para muestras independientes

Estadisticos de grupos

Control $X$

$\mathrm{N}=12$ media $=0,3050$ deviación típica $=$

0,01679 error tipo de la media $=0,00485$ 
ControlY

$\mathrm{N}=16$ media $=0,3081$ deviación típica $=$

0,01601 error tipo de la media $=0,00400$

Prueba de muestras independientes

Prueba de levene para la igualdad de varianzas

$F=0,037$ Sig. $=0,849$

Prueba T para la igualdad de medias

$\mathrm{T}=-0501 \mathrm{gl}=26 \mathrm{sig}$. (bilateral) $=0,621$

diferencias de medias $=-0,00313$

Error tipo de la diferencia $=0,00624$

$95 \%$ intervalo de confianza para la

diferencia

Superior $=-0,01595$ inferior $=0,00970$

Los resultados del contraste de la $\mathrm{T}$ con no significatividad al $99 \%$ para la diferencia de calidades en media ( $p$-valor mayor que alfa= 0,01 ) y con un intervalo de confianza para la diferencia de medias que evidentemente contiene el valor cero. No existe diferencia de calidad en media en la producción de leche en ambas granjas.

Problema 7: El gerente de compras de una empresa está considerando 4 máquinas de marcas diferentes para su uso en el ensamble de un producto particular. Estas están siendo comparadas con respecto a su velocidad. En un experimento diseñado para determinar si hay diferencias en la velocidad promedio de las 4 máquinas se observan los tiempos empleados en producir 6 articulos en forma aleatoria de cada máquina. Los tiempos registrados en segundos se presentan en la Tabla 03. Determine al nivel de significación del $5 \%$ si las máquinas llevan a cabo la tarea a la misma velocidad promedio

Tabla 03: tiempos en segundos empleados en la producción

Máquina

1. $55,46,45,73,50,63$,

$2.60,58,68,58,63,52$

$3.64,62,51,57,65,68$

$4.42,45,52,44,42,56$

Se realizará una prueba de análisis de varianza de un factor para muestras de tamaños iguales en la cual vamos a probar que la hipótesis nula los promedios poblacionales de las 4 máquinas son iguales.

\begin{tabular}{|l|r|r|r|r|r|}
\hline $\begin{array}{l}\text { Fuente de } \\
\text { variación }\end{array}$ & $\begin{array}{l}\text { Suma de } \\
\text { cuadrados }\end{array}$ & gl & $\begin{array}{r}\text { Media } \\
\text { cuadrática }\end{array}$ & F & Sig. \\
\hline Maquinas & 754,125 & 3 & 251,375 & 4,579 & .013 \\
Error & 1097,833 & 20 & 54,892 & & \\
Total & 1851,958 & 23 & & & \\
\hline
\end{tabular}

Se rechaza la hipótesis nula, ya que el valor de $\mathrm{p}$-valor es 0,013 menor que alfa $=$ 0,05 ; por lo tanto, se debe rechazar Ho aceptando que no todas las medias son iguales.

Problema 8: Cuatro profesores enseñan a cuatro secciones un mismo curso de Estadistica. Las calificaciones se registran en la Tabla 4. Al nivel de significación del $5 \%$, ¿se puede concluir que existe una diferencia significativa en las calificaciones promedio obtenidas con los cuatro profesores?

\section{Tabla 04:}

Profesores

P1. $12,11,9,10$

P2. $14,16,13,18,17,12$

P $3.13,12,9,11,12$

P4.10,17, 15, 14, 15

Se realizará una prueba de análisis de varianza de un factor para muestras de tamaños diferentes en la cual vamos a probar que la hipótesis nula los promedios poblacionales de las 4 calificaciones son iguales.

\begin{tabular}{|l|r|r|l|r|r|}
\hline & $\begin{array}{r}\text { Suma de } \\
\text { cuadrados }\end{array}$ & gl & $\begin{array}{l}\text { Media } \\
\text { cuadrática }\end{array}$ & F & Sig. \\
\hline Profesores & 69,000 & 3 & 23,000 & 5,33 &, 010 \\
Error & 69,000 & 16 & 4,313 & & \\
Total & 138,000 & 19 & & & \\
\hline
\end{tabular}

Se rechaza la hipótesis nula, ya que el valor de $p$-valor es 0,010 menor que alfa $=$ 0,05 ; por lo tanto, se debe rechazar Ho aceptando que no todas las medias de las calificaciones son iguales.

Problema 9: Del problema 8 la decisión fue rechazar la hipótesis nula de que las 4 medias de grupos son iguales. Luego, existen diferencias significativas entre las marcas de las maquinas en cuanto a la velocidad promedio para producir el articulo. Al nivel de significación del $5 \%$ y utilizando el método DMS, ¿ qué pares de medias son significativamente diferentes?

Se realizará una prueba de comparación múltiple a posteriori.

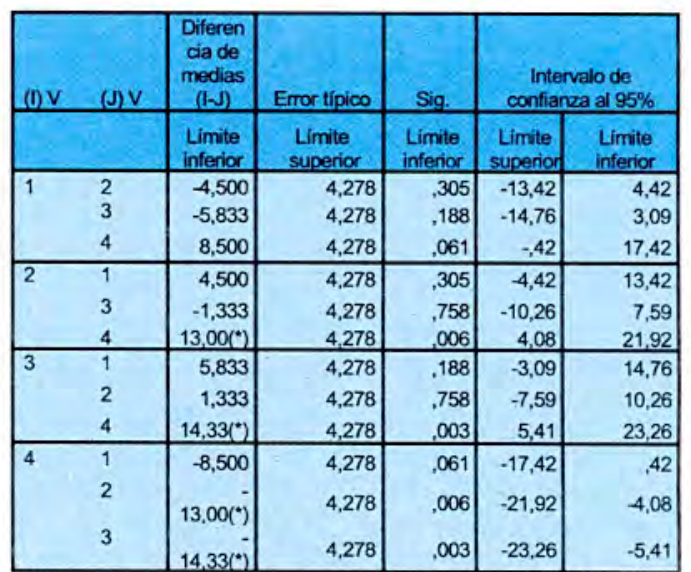

* La diferencia de medias es significativa al nivel.05.

Si las muestras tienen el mismo tamaño las diferencias de medias, en valor absoluto para todo i diferente de $\mathrm{j}$ se pueden resumir en comparaciones de pares de medias en filas y columnas. 
Se indican con * las diferencias significativas al $5 \%$. En este caso se tiene que 2 de los 6 pares de medias poblaciones son significativamente diferentes: u2 diferente de u4, y u3 diferente u4. Si las muestras no tienen el mismo tamaño, no existe un valor crítico único para realizar el contraste de pares de medias. En este caso se deben evaluar todos los posibles pares de medias en forma individual por la prueba t- de student.

Problema 10: Aplicar el método a posteriori de scheffe al problema 8.

Variable dependiente: Máquinas Scheffé La diferencia de medias es significativa al nivel 0,05 .

Aplicando el método de Scheffé se encuentra que solo la diferencia de los promedios 3 y 4 es significativa, luego se concluye por el método de Scheffé que u4 es diferente de u3.

Problema 11:Los valores sobre las longitudes en micras de 50 filamentos de la producción de una máquina ¿se supone normal? Son los siguientes:

$102,100,128,108,105,98,86,99,113,106,93,9$ $5,119,106,100,103,128,105,98,105,110,120$, $105,92,130,106,115,99,112,110,110,134,114$ $, 100,99,116,106,106,120,118,114,117,115,8$ $9,100,109,130,102,116,108$.

a) Hallar un intervalo de confianza para la media de la producción basado en la muestra de los 50 filamentos al $90 \%$ de coeficiente de confianza.

b) Contrastar la hipótesis nula de que la longitud media de los filamentos de la producción es de 100 al nivel de significancia del $10 \%$, basándose en la muestra dada.

Se trata de contrastar una prueba t para una muestra:

Filamento $\mathrm{N}=50$ media $=1083.8$ desviación típica $=10,81$

Prueba para una muestra

Valor de prueba $=100$

$\mathrm{T}=5,482, \mathrm{gl}=49$, sig. (Bilateral) $=0,00$

Diferencia de medias $=8,380$

Intervalo de confianza para la diferencia del $90 \%$

Inferior $=5,82$ superior $=10,94$

\begin{tabular}{|c|c|c|c|c|c|}
\hline Archivo & Edición & Ver & \multicolumn{2}{|c|}{ Datos } & \multirow{2}{*}{$\begin{array}{l}\text { Tre } \\
\text { 핖 }\end{array}$} \\
\hline$\otimes H$ & 国 & 판 & 4 & $\Leftrightarrow$ & \\
\hline \multicolumn{6}{|l|}{$3=$} \\
\hline 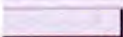 & & 1 & & $\sqrt{2}$ & \\
\hline 1 & & 57 & & & 55 \\
\hline 2 & & 49 & & & 48 \\
\hline 3 & & 60 & & & 58 \\
\hline 4 & & 55 & & & 56 \\
\hline 5 & & 57 & & & 54 \\
\hline 6 & & 48 & & & 48 \\
\hline 7 & & 50 & & & 52 \\
\hline 8 & & 61 & & & 56 \\
\hline 9 & & 52 & & & 50 \\
\hline 10 & & 56 & & & 58 \\
\hline
\end{tabular}
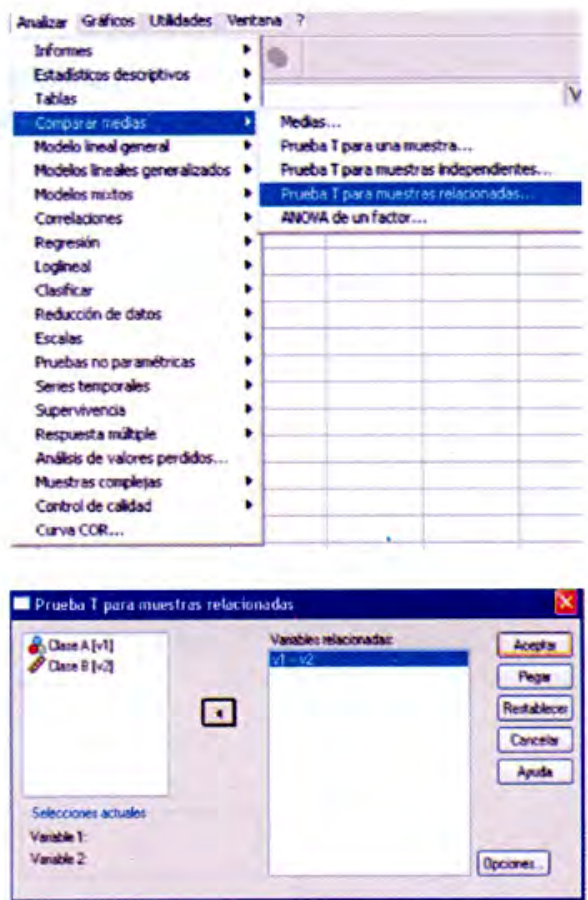

Prueba T para muestras relacionadas: Opciones X

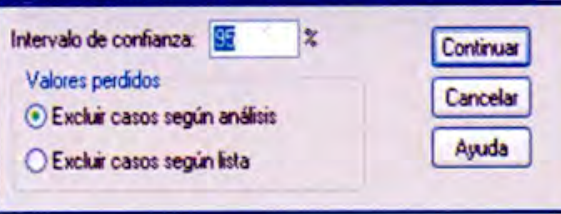

Estos gráficos muestran cómo se sigue el desarrollo de los problemas con SPSS

\section{DISCUSIONN}

El contraste de Kolmogorov-Smirnov se aplica solo a variables continuas y trata de medir el ajuste entre la función de distribución empírica de una muestra y la función de distribución teórica.

La diferencia máxima en valor absoluto de la frecuencia observada y esperada es 0,163 .

Por otra parte; en la tabla de valores de Kolmogorov- Smirnov para el nivel de significancia $\quad=0,05$ y $n=36$ se encuentra el valor crítico 0,221 . dado que $0,163<0,221$, debemos aceptar la hipótesis nula de que es normal la población de la cual se ha obtenido la muestra.

El test de Kruskal-Wallis es una versión no paramétrica del test $\mathrm{F}$ para comparar las medias de $\mathrm{k}$ poblaciones normales con varianza común. Este test contrasta la hipótesis nula de que $\mathrm{k}$ muestras provienen de la misma población.

Esta prueba de bondad de ajuste compara las frecuentas observadas y esperadas en cada categoria para contrastar si toda las categorias contienen la misma proporción de valores o si cada categoría contiene una proporción de valores especificado por el investigador.

Por lo tanto, se concluye que $p=0,548$ es mayor que $=0,01$ indicando que hay 
independencia entre color de cabellos y de ojos con un nivel de confianza del $99 \%$.

El procedimiento prueba $T$ para muestras relacionadas compara las medias de dos variables de un solo grupo. Calcula las diferencias entre los valores de las dos variables de cada caso y contrasta si la media difiere de 0 .

Entonces se observa que resulta que la diferencia de producciones medias para ambas clases de trigo no es significativa al 95\% ( $p$-valor del contraste de diferencia de medias mayor que $=0,05$ ) y lógicamente el intervalo de confianza para dicha diferencia $-0,617,2,617$ contiene el valor cero. Se puede concluir que las producciones son iguales en media para ambas clases de trigo.

El procedimiento prueba $\mathrm{T}$ para muestras independientes compara las medias de dos grupos de casos. Para esta prueba, idealmente los sujetos deben asignarse aleatoriamente a dos grupos, de forma que cualquier diferencia en la respuesta sea debida al tratamiento (o falta de tratamiento) y no a otros factores.

Entonces los resultados del contraste de la T con no significatividad al $99 \%$ para la diferencia de calidades en media ( $p$-valor mayor que = 0.01) y con un intervalo de confianza para la diferencia de medias que evidentemente contiene el valor de cero. No existe diferencia de calidad en media en la producción de leche de ambas granjas.

El análisis de varianza es una técnica estadistica para comprobar si son iguales las medias de más de dos poblaciones mediante el análisis y la comparación de diversos tipos de varianzas muestrales insesgadas.

Observamos que el valor $p=0,013$ es menor que alfa $=0,05$ aceptando que no todas las medias son iguales. Pero cuando aplicamos la prueba de comparación múltiple a posteriori se indican las diferencias significativas al 5\%. En este caso se tiene que dos de los seis pares de medias poblacionales son significativamente diferentes.

Aplicando el método de Scheffé se encuentra que solo la diferencia de los promedios de la máquina 3 y 4 es significativa, concluyendo que la media poblacional de la máquina 4 es diferente de la máquina 3.

En la prueba para una muestra para probar la media de la poblacional en la cual la prueba t se rechaza la hipótesis nula por $p$ es mayor que alfa 0,10

\section{CONCLUSIONES}

1.Las pruebas estadísticas paramétricas se aplican principalmente a datos de tipo cuantitativo y cada una de ellas exige el cumplimiento de algunos supuestos, de los cuales el que aparece en la mayor parte de las pruebas se refiere a la normalidad de la población de la cual fue extraída la muestra.
2.Las pruebas estadisticas no paramétricas no dependen de la distribución que puedan adoptar los datos. Se dice que una prueba es robusta si sus resultados son válidos incluso cuando los datos se apartan sensiblemente de las condiciones teóricas.

3.Los procedimientos estadísticos no paramétricos descritos buscan ayudar en la planificación, conducción e interpretación de experimentos.

4.Sea cual sea el contraste que utilicemos, la hipótesis nula traducirá la idea de que en los diferentes grupos se obtienen resultados similares, y la hipótesis alternativa lo negará. La significación del contraste nos dará una idea de si las diferencias observadas en los diferentes grupos son imputables al azar (significación grande) o hay una diferencia intrínseca entre algunos grupos (significación pequeña).

\section{RECOMENDACIONES}

Hacer la prueba de la normalidad en la estadistica parametrica no suponer que tiene una distribucion normal.

Aplicar problemas reales usando la estadistica no parametrica.

\section{BIBLIOGRAFIAA}

Ávila Acosta R.B. (1998). Estadistica Elemental. Lima .

Canavos G,C. (2003). Probabilidad y Estadistica.: Aplicaciones y Métodos.Mc Graw Hill.

Córdoba Zamora Manuel (1999). Estadistica Inferencial, Lima.

Freund J. E., Millar, I; y Millar, M.; (2000). "Estadistica Matemáticas con Aplicaciones."

Moya Calderón, Rufino. (1998). Estadística Descriptiva. Conceptos y aplicaciones. Lima.

Pardinas, Felipe. (1972).Metodología y Técnicas de Investigación en Ciencias Sociales. México. Siglo XXI Editores.

Ruiz Maya, L. (2000). Métodos Estadisticos de Investigación en las Ciencias Sociales: Técnicas no Paramétricas.

Torres Bardales, C. (1998) Metodologia de la Investigación Científica.Lima.

Tamayo Calderón, José. (1993).Fundamentos de la Investigación Científica en los servicios Médicos. UNMSM .Lima.

Wayne W., Daniel. (1995). Bioestadística. Editorial Limusa, S:A: de C.V. México.

Pérez César (2001). Técnicas Estadisticas con SPSS. Editorial Isabel Capella. Madrid 